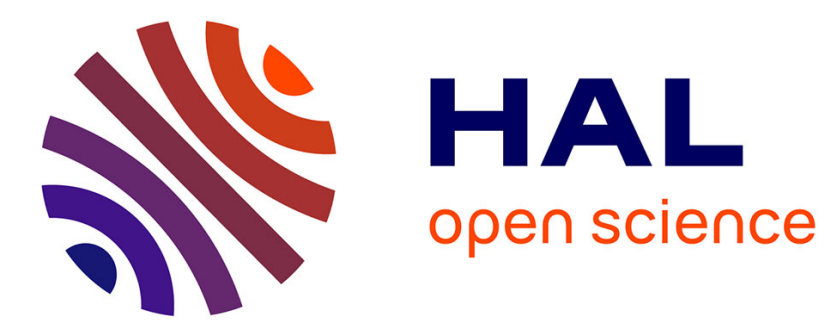

\title{
Temperature-dependent anhysteretic behavior of co-doped PZT
}

Valentin Segouin, Barbara Kaeswurm, Kyle G. Webber, Laurent Daniel

\section{To cite this version:}

Valentin Segouin, Barbara Kaeswurm, Kyle G. Webber, Laurent Daniel. Temperature-dependent anhysteretic behavior of co-doped PZT. Journal of Applied Physics, 2018, 124 (10), pp.104103. 10.1063/1.5040556 . hal-01943246

\section{HAL Id: hal-01943246 https://hal.science/hal-01943246}

Submitted on 16 Jul 2020

HAL is a multi-disciplinary open access archive for the deposit and dissemination of scientific research documents, whether they are published or not. The documents may come from teaching and research institutions in France or abroad, or from public or private research centers.
L'archive ouverte pluridisciplinaire $\mathbf{H A L}$, est destinée au dépôt et à la diffusion de documents scientifiques de niveau recherche, publiés ou non, émanant des établissements d'enseignement et de recherche français ou étrangers, des laboratoires publics ou privés. 


\section{Temperature-dependent anhysteretic behavior of co-doped PZT}

Cite as: J. Appl. Phys. 124, 104103 (2018); https://doi.org/10.1063/1.5040556

Submitted: 18 May 2018 . Accepted: 23 August 2018 . Published Online: 13 September 2018

Valentin Segouin, Barbara Kaeswurm (D, Kyle G. Webber, and Laurent Daniel
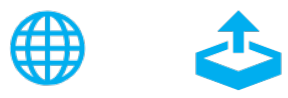

\section{ARTICLES YOU MAY BE INTERESTED IN}

$\mathrm{BaTiO}_{3}$-based piezoelectrics: Fundamentals, current status, and perspectives Applied Physics Reviews 4, 041305 (2017); https://doi.org/10.1063/1.4990046

Enhanced thermal stability of dielectric, energy storage, and discharge efficiency in a structurally frustrated piezoelectric system: Erbium modified $\mathrm{Na}_{0.5} \mathrm{Bi}^{-} ._{5} \mathrm{TiO}_{3}-\mathrm{BaTiO}_{3}$ Journal of Applied Physics 124, 104101 (2018); https://doi.org/10.1063/1.5035263

Direct observation of the domain kinetics during polarization reversal of tetragonal PMN-PT crystal

Applied Physics Letters 113, 112902 (2018); https://doi.org/10.1063/1.5046657

\section{Lock-in Amplifiers up to $600 \mathrm{MHz}$}
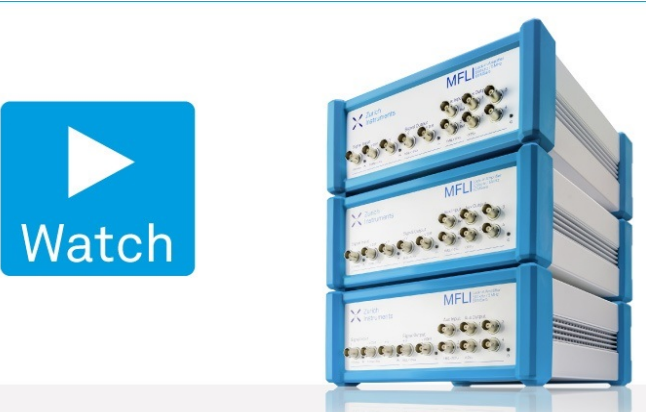


\title{
Temperature-dependent anhysteretic behavior of co-doped PZT
}

\author{
Valentin Segouin, ${ }^{1}$ Barbara Kaeswurm, ${ }^{2}$ Kyle G. Webber, ${ }^{2}$ and Laurent Daniel ${ }^{1}$ \\ ${ }^{1}$ GeePs $\mid$ Group of Electrical Engineering-Paris, UMR CNRS 8507, CentraleSupélec, Univ. Paris-Sud, \\ Université Paris-Saclay, Sorbonne Université, 3 \& 11 rue Joliot-Curie, Plateau de Moulon, \\ 91192 Gif-sur-Yvette CEDEX, France \\ ${ }^{2}$ Materials Science and Engineering Department, Friedrich-Alexander-Universität Erlangen-Nürnberg, \\ 91058 Erlangen, Germany
}

(Received 18 May 2018; accepted 23 August 2018; published online 13 September 2018)

The anhysteretic behavior of a soft $\mathrm{Pb}(\mathrm{Zr}, \mathrm{Ti}) \mathrm{O}_{3}$ was measured from $25^{\circ} \mathrm{C}$ to $175^{\circ} \mathrm{C}$. The experimental determination of the anhysteretic polarization curve, combined with classical $P$ - $E$ and $S$ $E$ loop measurements, allows for an experimental separation of the reversible and dissipative contributions to the ferroelectric behavior. This approach offers insight into the different mechanisms originating at the microscopic scale and the contribution to the macroscopic ferroelectric properties. It was found that the reversible anhysteretic susceptibility $\chi_{a}$ of the unpoled material increases by $30 \%$ from room temperature to $150^{\circ} \mathrm{C}$. On the other hand, the effect on the total susceptibility for a null polarization $\chi_{c}$ increases only by $17 \%$ over the same temperature range. Since the difference between $\chi_{a}$ and $\chi_{c}$ reflects the dissipative contribution to the macroscopic ferroelectric behavior, this reveals that dissipation reduces the improvement of susceptibility under increasing temperature. This work illustrates the benefits of separating experimentally the reversible and dissipative contributions to describe the ferroelectric behavior, which can serve as a basis for advanced modeling approaches. Published by AIP Publishing. https://doi.org/10.1063/1.5040556

\section{INTRODUCTION}

Ferroelectric materials are widely used in electronic applications, such as tunable capacitors, non-volatile memory, oscillators, and filters, as well as transduction applications, such as sensors and actuators. During operation, these materials can be subjected to significant heat due to thermal dissipation (Joule effect) or when the application is used in a harsh environment. The temperature can vary from $-20^{\circ} \mathrm{C}$ to $85^{\circ} \mathrm{C}$ in typical electronic devices and sometimes within a much larger range, like in military applications. ${ }^{1,2}$ The typical temperature range for piezoelectric actuators in fuel injection systems, for example, is from $-55^{\circ} \mathrm{C}$ to $150^{\circ} \mathrm{C} .{ }^{3}$ Such temperature variations can significantly change the electromechanical behavior of ferroelectric materials through variations in both the intrinsic and extrinsic contributions. The characterization of ferroelectrics under temperature is, therefore, a key point for the design of engineered devices that use ferroelectrics. For instance, a strong nonlinear coupling between the dielectric constant and the temperature was observed in Hooker's work. ${ }^{4}$ Hooker further showed that the coercive field and the remanent polarization of $\mathrm{Pb}(\mathrm{Zr}, \mathrm{Ti}) \mathrm{O}_{3}$ (PZT) significantly decrease from $0{ }^{\circ} \mathrm{C}$ to $250^{\circ} \mathrm{C}$. These strong couplings are not limited only to electrical properties. The piezoelectric and elastic properties of PZT are also highly affected by temperature changes. In the work of Kaeswurm et al., ${ }^{5}$ significant nonlinear variations in the piezoelectric coefficient $d_{33}$ were found from -150 to $250^{\circ} \mathrm{C}$.

There have been a number of other similar investigations on the temperature-dependence of ferroelectrics. ${ }^{6,7}$ Despite this, however, the influence of temperature on ferroelectrics remains challenging due to a complex thermoelectromechanical coupling. Indeed, the ferroelectric behavior (polarization $P$ or strain $S$ as a function of applied electric field $E$ ) is the result of different intrinsic and extrinsic mechanisms. ${ }^{89}$ The intrinsic behavior corresponds to the piezoelectric behavior, also called "volume contribution." It is the lattice contributions that would be observed in a single ferroelectric domain. The extrinsic behavior refers to the hysteretic ferroelectric mechanisms, which correspond to domain switching and domain wall vibration, referred to as macrohysteresis and microhysteresis by Lewis. ${ }^{10}$ Similarly, the motion of phase boundaries ${ }^{11}$ and polarization rotation ${ }^{12}$ are also possible. The extrinsic effects contribute to the ferroelectric reversible behavior but are also associated with significant energy dissipation, directly resulting in the typical hysteresis of the $P-E$ and $S-E$ loops observed in ferroelectrics.

A way to simplify the study of ferroelectric behavior is to separate the reversible and dissipative contributions to the macroscopic behavior. Such separation was already conducted in the past for a sub-coercive field using Rayleigh analysis. ${ }^{13-16}$ These studies helped to distinguish the reversible and irreversible domain wall motion around a metastable equilibrium position. Another approach is to separate dissipative phenomena as a whole from the reversible part of the behavior. This reversible part is associated with the absolute equilibrium of domain walls, without introducing the role of domain wall pinning. We have recently shown ${ }^{17}$ that the reversible part of ferroelectric behavior can be experimentally reconstructed by determining the anhysteretic behavior. In analogy to the anhysteretic curve for ferromagnetic materials, ${ }^{18}$ a reversible $P$ - $E$ curve is experimentally determined through the application of a bipolar electric field with a decaying maximum electric field, resulting in a so-called anhysteretic curve or an ideal polarization curve. The anhysteretic curve is the representation of the reversible part of the 
material behavior that reflects the state of the microstructure in the absence of dissipation effects, such as friction, domain switching, domain wall pinning, and dielectric losses. In the case of hysteretic behavior, domain walls are constrained by internal forces (pinning on defects, friction effects at domain, and grain boundaries). Due to these forces, a threshold energy must be overcome to move from a given domain configuration to another. This leads to the typical hysteretic shape of $P-E$ loops. In the case of anhysteretic behavior, for a given level of electric field, these internal forces are overcome by providing the material with sufficient energy to ensure that domain walls can reach their absolute equilibrium state, with no impediment due to defects. The anhysteretic curve then represents the behavior of the material as if domains could expand and reduce freely: the behavior is still non-linear but reversible, meaning that it shows no dissipation.

Such a representation of the ferroelectric behavior as the sum of reversible and dissipative contributions can be of great help to develop modeling tools for ferroelectric materials. ${ }^{19}$ Importantly, however, the temperature dependence of anhysteretic ferroelectric behavior has never been investigated, despite the significant effects on the ferroelectric mechanisms induced by temperature, as discussed by Hall. ${ }^{20}$ Herbiet et al., ${ }^{21}$ for instance, observed a $40 \%$ decrease in the reversible extrinsic contribution to the permittivity from 0 to $150{ }^{\circ} \mathrm{C} .{ }^{21}$ In another work from Zhang et al., ${ }^{22}$ a variation of $0.7 \% /{ }^{\circ} \mathrm{C}$ was observed on the extrinsic permittivity around $0{ }^{\circ} \mathrm{C}$. ${ }^{22}$ Such temperature effects have also been observed on the piezoelectric constant. In a study on a PIN-PMN-PT single crystal, Schader et al. ${ }^{23}$ found that the intrinsic contribution to $d_{33}$ quadruples from room temperature to $100^{\circ} \mathrm{C}$. In addition to this, the intrinsic $d_{33}$ at room temperature was found to represent $80 \%$ of the total piezoelectric constant in single crystal PZN-PT. ${ }^{24}$

In this work, for the first time, the anhysteretic behavior of a ferroelectric ceramic is studied at elevated temperatures. The protocol and the experimental setup used for the measurements are presented in a first part; details are given to answer technical issues associated with the measurements. In a second part, the temperature dependence of the behavior is measured through classical $P-E$ and $S-E$ loops as well as the $P-E$ anhysteretic curve. Using post-treatment processes, the $S-E$ anhysteretic curve is obtained. A set of parameters are then defined, and their evolution as a function of temperature is discussed. The initial slope of the anhysteretic curve, which is referred to as the anhysteretic susceptibility $\chi_{a}$, is determined as a function of temperature. Information on the contribution of the dissipation mechanism on the overall macroscopic behavior is obtained via a detailed comparison of the conventional susceptibility $\chi_{c}$. The results show how the changes in behavior due to elevated temperature can be distributed between the different ferroelectric mechanisms.

\section{EXPERIMENTAL METHODOLOGY}

The material investigated was the commercially available polycrystalline $\mathrm{Pb}_{0.990}\left(\mathrm{ZrTi}_{0.47}\left(\mathrm{Sb}_{0.67} \mathrm{Nb}_{0.33}\right)_{0.08}\right) \mathrm{O}_{3}$ (PIC 151, PI Ceramics). The samples were ground to the final dimensions of $5 \mathrm{~mm} \times 5 \mathrm{~mm} \times 1 \mathrm{~mm}$ parallelepipeds using a surface grinder. Gold electrodes were sputtered onto opposing $5 \mathrm{~mm} \times 5 \mathrm{~mm}$ surfaces. Prior to measurement, each sample was thermally depolarized by heating up to $600^{\circ} \mathrm{C}$ and then cooling down to room temperature at a rate of $100^{\circ} \mathrm{C} / \mathrm{h}$. The electric displacement was measured during experimentation with a modified Sawyer-Tower circuit $^{25}$ that consisted of an integrated reference capacitor $(4.67 \mu \mathrm{F})$ and a 1:1 operational amplifier. The uniaxial strain was obtained from a linear variable differential transformer sensor (HBM 1-WA/2 MM-T) and from the data acquisition system HBM MGCplus AB22A. Voltage was applied with a high voltage amplifier (20/20C, TREK Inc.) controlled by either a function generator (HP 33120A, Agilent Technologies Inc.) or a LabVIEW program via an analog output device (NI PCI-6221, National Instruments Corp.). The same LabVIEW program was also used for data acquisition and analysis, i.e., electric field, strain, and electric displacement. During testing the sample was immersed in a temperature-controlled silicone oil (WACKER AK 200, Wacker Chemie AG) bath (Haake 30P) to prevent electrical arcing. During experiments, the silicone oil was heated and cooled by an active temperature control system integrated into the bath with a resolution of $\pm 0.1^{\circ} \mathrm{C}$.

Because defects cannot be fully eliminated from the material, it is not possible to directly determine the anhysteretic curve from the typical bipolar electric field loading. Such bipolar loadings are well known and result in the classical $P$-E loop with the corresponding hysteresis. The anhysteretic behavior, however, can be reconstructed following a specific experimental procedure. The method, referred to as the bias field method, has been detailed in our recent paper. ${ }^{17}$ In this method, the material is loaded by an electric field $E(t)$ composed of an alternating decaying component and a bias field component [see Eq. (1)]. At the end of this electrical loading process, the domain state has reached a stable equilibrium, which is independent from the dissipation sources. The following equation was used to apply a decaying electric field to the sample during testing at various bias electric field levels:

$$
E(t)=E_{\text {max }} \sin (\omega t) \exp (-k \omega t)+E_{\text {bias }}[1-\exp (-k \omega t)]
$$

where $E_{\max }$ is the maximum amplitude of the alternating field, $\omega$ is the angular frequency of the alternating field $(\omega$ $=\frac{f}{2 \pi}$ with $f$ the frequency), $t$ is time $\left(t \in\left[0 ; t_{\max }\right]\right.$ with $t_{\text {max }}$ the duration of the measurement), $k$ is the exponential decay constant (or damping factor), and $E_{\text {bias }}$ is the amplitude of the bias field.

During testing, the ferroelectric sample was electrically loaded, as described by Eq. (1). For $t \geq t_{\max }$, the remaining applied field is a constant $E_{\text {bias }}$, where the corresponding electric displacement was found to stabilize to an equilibrium value $D_{a}$. The measurement of the couple $\left(E_{\text {bias }}, D_{a}\right)$ provides one point of the anhysteretic curve. The full sequence is then repeated for different $E_{\text {bias }}$ values to construct the full anhysteretic curve. For this reason, an anhysteretic curve cannot be measured in one continuous sweep. It is important to note that the anhysteretic electric 
displacement $D_{a}$ is not strictly independent of the selected loading parameters, i.e., $E_{\max }, f, k$, and $t_{\max }$. The independence can nevertheless be assumed when: (i) the maximum field amplitude is high enough so that the technical saturation is reached before the exponential decay towards the bias field, which ensures that dissipation effects are overcome whatever the initial equilibrium position; (ii) the frequency is low enough to be considered quasi-static as the influence of defects on domains alignment is not negligible for dynamic loadings; (iii) the decay constant is sufficiently small to prevent domain walls to stop on a metastable equilibrium; and (iv) the duration of the anhysteretic sequence is long enough to have a negligible polarization hysteresis at the end of the experiment. The effect of $E_{\max }, f$, and $k$ on the anhysteretic polarization was investigated for PIC151. It was found that for $E_{\text {bias }} \leq 2 \mathrm{kV} / \mathrm{mm}$, the following conditions ensured the repeatability of the measurements: $E_{\max } \geq 3 \mathrm{kV} / \mathrm{mm}$, $f \leq 0.5 \mathrm{~Hz}, t_{\max }>60 \mathrm{~s}$, and $k \leq 0.015$.

As previously noted, the polarization hysteresis at the end of the experiment must be negligible to correctly measure $D_{a}$. In other words, variations of $E(t)$ around $t_{\max }$ must be small and thus, $t_{\max }$ must be set high enough. However, the $t_{\max }$ value cannot be set arbitrarily high due to the sample conductivity. Although the current flowing through the sample does not contribute to its polarization, it appears as a time-dependent drift in electric displacement due to charge accumulation on the reference capacitor in the SawyerTower circuit. While this conduction is generally low and can, therefore, be neglected at low temperatures, an increase in the conductivity of PZT at high temperature ${ }^{26}$ can influence the measurements. A way to limit the apparent electric displacement drift is to limit the experiment duration $t_{\max }$. In this work, conductivity effects were investigated (see Appendix A) and the study revealed that $t_{\max }$ should be less than $60 \mathrm{~s}$ to keep the polarization drift under $2 \%$ of the major

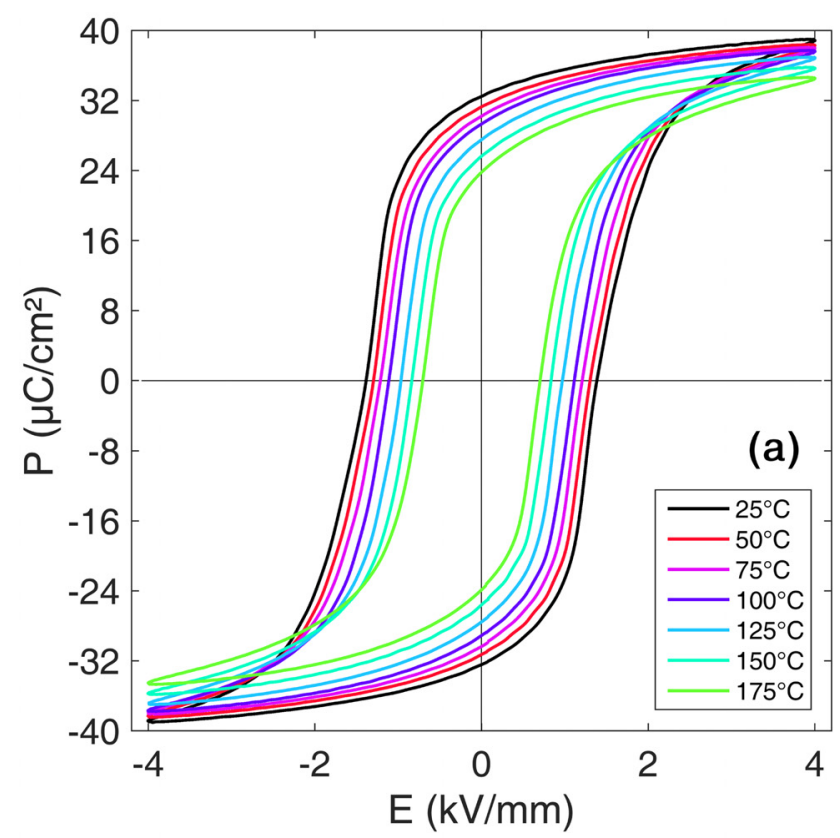

loop amplitude. Given the chosen parameters, the amplitude in polarization of the last pseudo-period does not exceed $1 \%$ of the major loop amplitude, which is acceptable. Duration of $60 \mathrm{~s}$ is then adopted for all experiments. For $E_{\max }>1 \mathrm{kV} /$ $\mathrm{mm}$ and $\mathrm{T}>150^{\circ} \mathrm{C}$, the duration needs to be reduced because of a higher conductivity. For the four corresponding points, $t_{\max }$ is progressively reduced to $20 \mathrm{~s}$.

\section{RESULTS}

The PZT sample was first analyzed by measuring the classical $P-E$ and $S-E$ hysteresis loops at various temperatures from $25^{\circ} \mathrm{C}$ to $175^{\circ} \mathrm{C}$ (Fig. 1). All the loops were measured using a sine waveform with a frequency of $0.5 \mathrm{~Hz}$ and a maximum electric field of $4 \mathrm{kV} / \mathrm{mm}$. The $P$-E loops were centered using the minimum and maximum polarization values [origin defined as $\left(P_{\max }+P_{\min }\right) / 2$ ]. The reference point between strain-electric field loops [Fig. 1(b)] was the remanent strain at zero electric field. With an increase in temperature, there is an apparent decrease in both the remanent polarization and the coercive field, both well-known phenomena in PZT. ${ }^{27}$ Despite this, however, the maximum strain was found to correspond well to previous temperaturedependent measurements on a similar composition. ${ }^{28}$

Following the measurement procedure outlined in Sec. II, the anhysteretic curve was characterized from $25^{\circ} \mathrm{C}$ to $175^{\circ} \mathrm{C}$ (Fig. 2). As the polarization is a relative measurement, three $P$ - $E$ loops were measured at $4 \mathrm{kV} / \mathrm{mm}$ before each anhysteretic measurement. The reference was then defined as $\left(P_{\max }+P_{\min }\right) / 2$ similar to $P$-E loops of Fig. 1. As expected, the anhysteretic curves are non-linear with a steep variation at low fields and saturation at high fields. In the material investigated here, the residual polarization at $E=0 \mathrm{kV} / \mathrm{mm}$ can reach up to $1 \mu \mathrm{C} / \mathrm{cm}^{2}$, which is approximately $2.5 \%$ of the maximum polarization. This is likely due

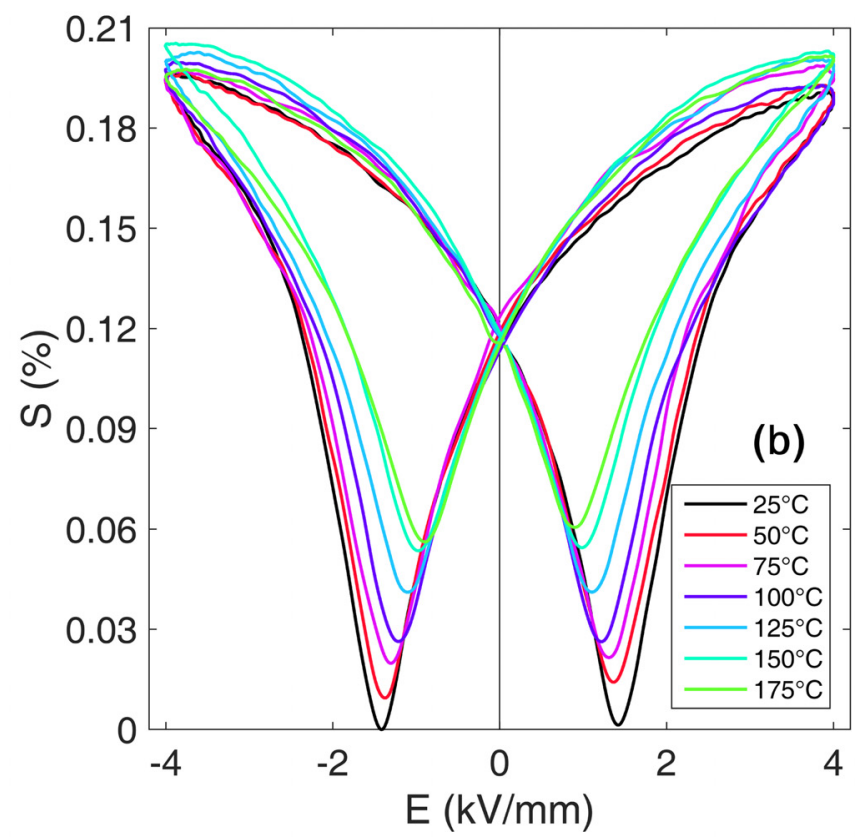

FIG. 1. (a) Polarization- and (b) strain-electric field hysteresis loops measured at various temperatures from $25^{\circ} \mathrm{C}$ to $175^{\circ} \mathrm{C}$. The applied electric field had a sinusoidal waveform at $0.5 \mathrm{~Hz}$. 


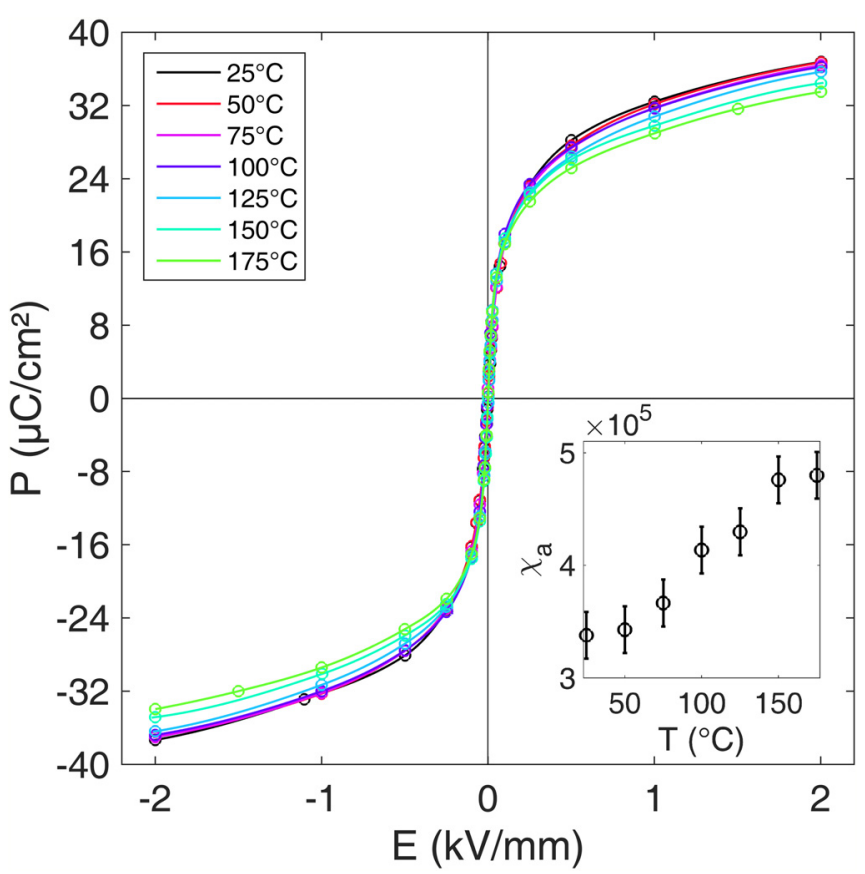

FIG. 2. Anhysteretic polarization curves measured at various temperatures between $25^{\circ} \mathrm{C}$ and $175^{\circ} \mathrm{C}\left(E_{\max }=4 \mathrm{kV} \mathrm{mm}^{-1} ; f=0.5 \mathrm{~Hz} ; t=60 \mathrm{~s}\right.$; $k=0.015)$. The inset shows the anhysteretic susceptibility at $E=0$ as a function of temperature.

to residual stresses or switching effects (due to domain wall pinning) inducing a residual polarization.

Similarly, it is also possible to simultaneously measure the anhysteretic $S$ - $E$ curve. In the present case, however, it was not possible to obtain a reliable measurement of the anhysteretic strain due to the measurement duration and external vibrations. For that reason, the anhysteretic strain was estimated by determining the electrostrictive behavior, similar to a previous study that used this dependence to characterize and model PZT as a function of temperature from

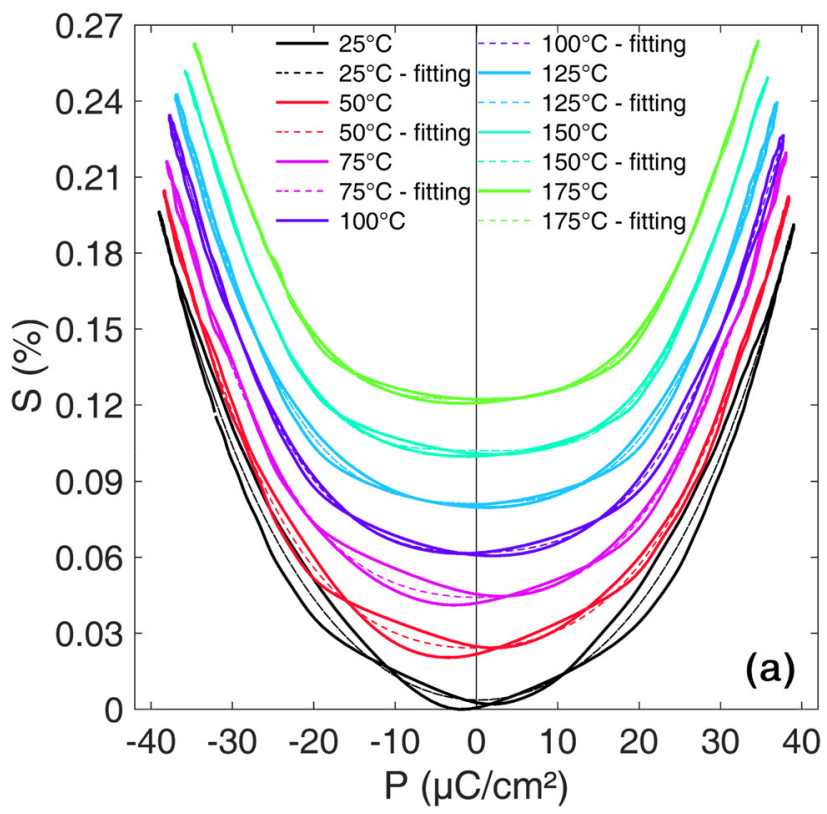

room temperature to $200{ }^{\circ} \mathrm{C}$. $^{29}$ It was found that the electrostrictive $S(P)$ relation gives a reliable representation of the ferroelectric behavior in this temperature range. In the present work, $S(P)$ is obtained for all temperatures by combining the electromechanical measurements from Fig. 1. Although the strain and polarization are measured simultaneously, a slight time phase-shift was observed between the two variables. This phase-shift was estimated by crosscorrelation between $S(t)$ and $P(t)$ and was found to increase with temperature, without exceeding $14 \mathrm{~ms}$ at $0.5 \mathrm{~Hz}$. For a given temperature, the phase-shift appeared to be constant as a function of time. As $S(t)$ was the delayed signal, the phenomenon was attributed to the time response of the LVDT sensor. $S(t)$ was then time-shifted to compensate for this phase difference. Figure 3(a) shows the resulting temperaturedependence of $S(P)$. $S(P)$ was fitted with a representative even polynomial function:

$$
S=\sum_{i=1}^{n} Q_{2 i-1} \cdot P^{2 i}(E),
$$

where $\mathrm{Q}$ is the electrostrictive coefficient.

In the present case, the $S(P)$ function is satisfactorily approximated using a 6 th order polynomial fitting $(n=3)$ for all temperatures. The estimated anhysteretic strain [Fig. 3 (b)] is then computed from the polynomial fittings and from the anhysteretic polarization curves. The anhysteretic strain exhibits a slight asymmetry for all temperatures (also visible on the $S$-E loops). At $25^{\circ} \mathrm{C}$, the difference between strains at $\operatorname{maximum}(E=2 \mathrm{kV} / \mathrm{mm})$ and minimum $(E=-2 \mathrm{kV} / \mathrm{mm})$ electric fields is about $0.01 \%$. This asymmetry comes from the anhysteretic polarization since there is a $0.8 \mu \mathrm{C} / \mathrm{cm}^{2}$ difference between values at maximum $(E=2 \mathrm{kV} / \mathrm{mm})$ and minimum electric field $(E=-2 \mathrm{kV} / \mathrm{mm})$. The data in Figs. 3(a) and 3(b) are then taken from Fig. 1, whereas the

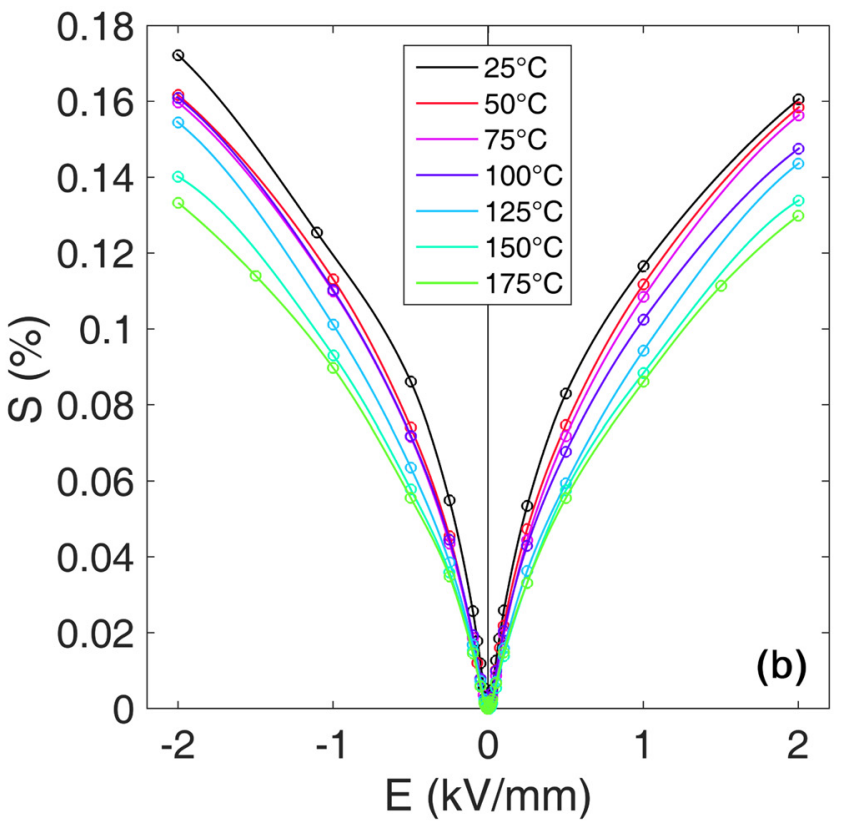

FIG. 3. (a) Strain-polarization dependence; a 6th order polynomial fitting is shown for each curve (dotted line). (b) Anhysteretic strain estimated from the strain-polarization dependence and from the anhysteretic polarization. 
asymmetry observed is explained by using Fig. 2. The polarization difference (Fig. 2) is attributed to a measurement error. As $0.8 \mu \mathrm{C} / \mathrm{cm}^{2}$ represents only $1.1 \%$ of the anhysteretic polarization curve amplitude (Fig. 2) and as a strain difference of $0.01 \%$ represents $6.8 \%$ of the anhysteretic strain amplitude [Fig. 3(b)], these errors were neglected.

\section{DISCUSSION}

During the electrical loading of the PZT sample, there is a change in the domain structure due to electric fielddependent domain wall motion. These domain walls encounter a network of defects, such as lattice vacancies and grain boundaries, which can act as pinning centers that restrict their motion and result in the observed hysteretic behavior. A representation of domain wall motion during such a process is described in Fig. 4(a). At $t=0$, the domain configuration is illustrated in image 1 , where the domain wall is located on the left side. From this configuration, $E(t)$ is applied to the material, resulting in irreversible domain wall motion past the defect network [represented in Fig. 4(a) by randomly positioned points]. During the initial oscillation of $E(t)$, the amplitude is large enough to move the domain wall past these various defects; no defect is strong enough to pin the domain wall. At this stage, the domain wall goes well beyond the initial equilibrium position, although it is oscillating around the equilibrium position with a vanishing amplitude. With a further decrease in the applied electric field amplitude, the domain wall will eventually reach its true equilibrium position for the given $E_{\text {bias }}$. The procedure can be repeated for different $E_{\text {bias }}$ levels, and the anhysteretic curve is then constructed in a discrete manner, as shown in Fig. 4(b). The parameters of the function $E(t)$ have to be meticulously determined in order to avoid the determination of false equilibrium positions. It was assumed in this study that the parameters were correct when a further refinement did not change the final measurement.
In order to discuss the material behavior in detail, four parameters were extracted from the polarization-electric field during bipolar and anhysteretic measurements: (i) the remanent polarization $P_{r}$, (ii) the coercive field $E_{c}$, (iii) the maximum polarization, and (iv) the anhysteretic susceptibility $\chi_{a}$ at $E=0 \mathrm{kV} / \mathrm{mm}$. The remanent polarization and the coercive field were obtained during electric field loading at $0.1 \mathrm{~Hz}$ with a maximum electric field of $2 \mathrm{kV} / \mathrm{mm}$ and $4 \mathrm{kV} / \mathrm{mm}$. Both parameters include dissipative and reversible effects. The maximum polarization was determined from the anhysteretic curve at $2 \mathrm{kV} / \mathrm{mm}$ (Fig. 2). This value is compared to the maximum polarization on $P-E$ loops at $0.1 \mathrm{~Hz}$ and $2 \mathrm{kV} / \mathrm{mm}$ maximum electric field. The final parameter, $\chi_{a}$, corresponds to the slope of the anhysteretic polarization curve at the origin. This parameter represents the material response in the absence of any dissipation mechanisms. Here, $\chi_{a}$ is computed using a simple linear regression of 6 anhysteretic points between -15 and $15 \mathrm{~V} / \mathrm{mm}$. Out of the range $\pm 15 \mathrm{~V} / \mathrm{mm}$, the anhysteretic curve becomes significantly non-linear.

The evolution of the remanent polarization and the coercive field as a function of temperature are shown in Fig. 5. It is found that both parameters decrease with temperature, which is in agreement with previous data reported for PZT. ${ }^{30}$ The observed decrease in the coercive field was found to be linear with a substantial rate, namely $-0.33 \% /{ }^{\circ} \mathrm{C}$ from the coercive field at room temperature. Such a decrease was expected since the Curie temperature of the material is about $250{ }^{\circ} \mathrm{C} .{ }^{27} \mathrm{~A}$ constant offset can be noticed between the negative and positive coercive field values $(10 \mathrm{~V} / \mathrm{mm}$ difference for $E_{\max }=4 \mathrm{kV} / \mathrm{mm}$ ). This is attributed to a measurement error and is neglected since it represents only $0.8 \%$ of the coercive field at $25^{\circ} \mathrm{C}$. The electric field amplitude $E_{\text {max }}$ was also observed to influence both the observed coercive field and the remanent polarization; between 2 and $4 \mathrm{kV} / \mathrm{mm}$, for example, there was a difference of $150 \mathrm{~V} / \mathrm{mm}$ of $E_{c}$ and

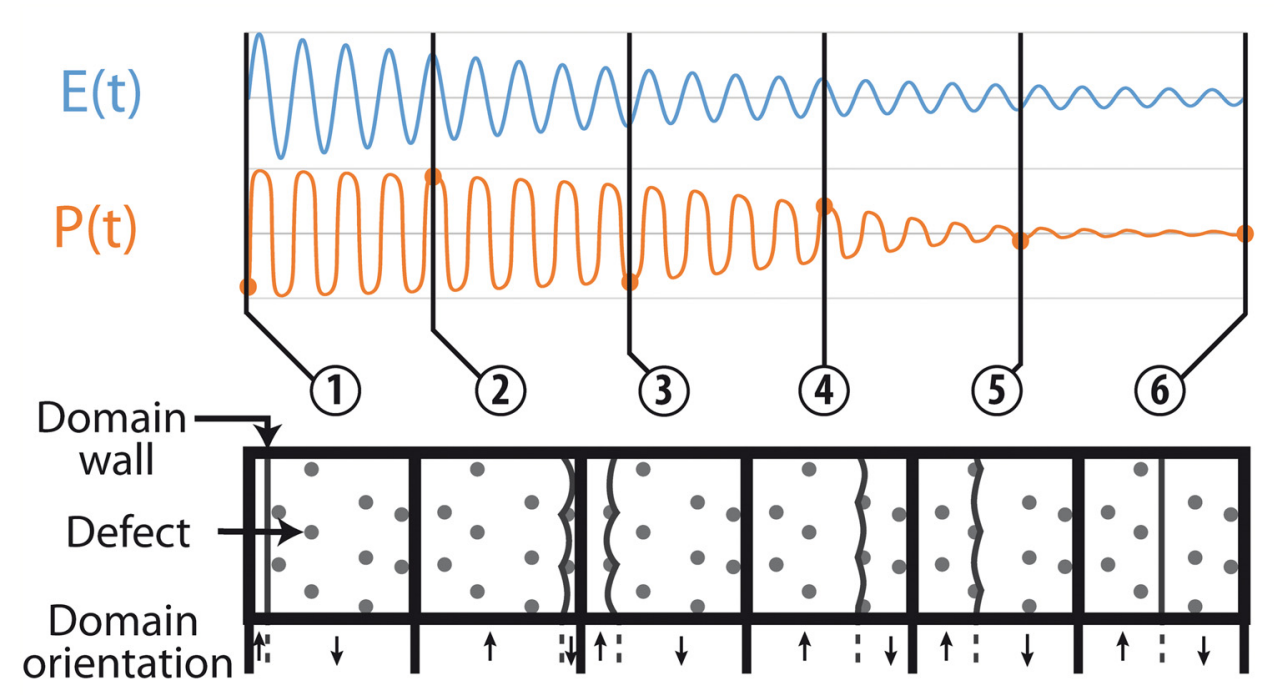

(a)

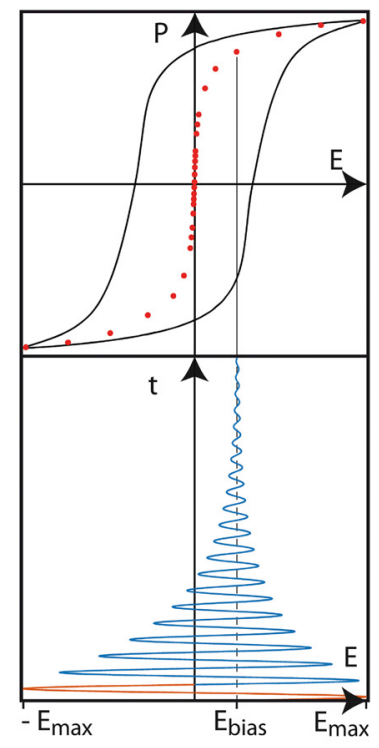

(b)

FIG. 4. (a) Illustration of a domain wall motion during this procedure and (b) illustration of the anhysteretic measurement procedure. 


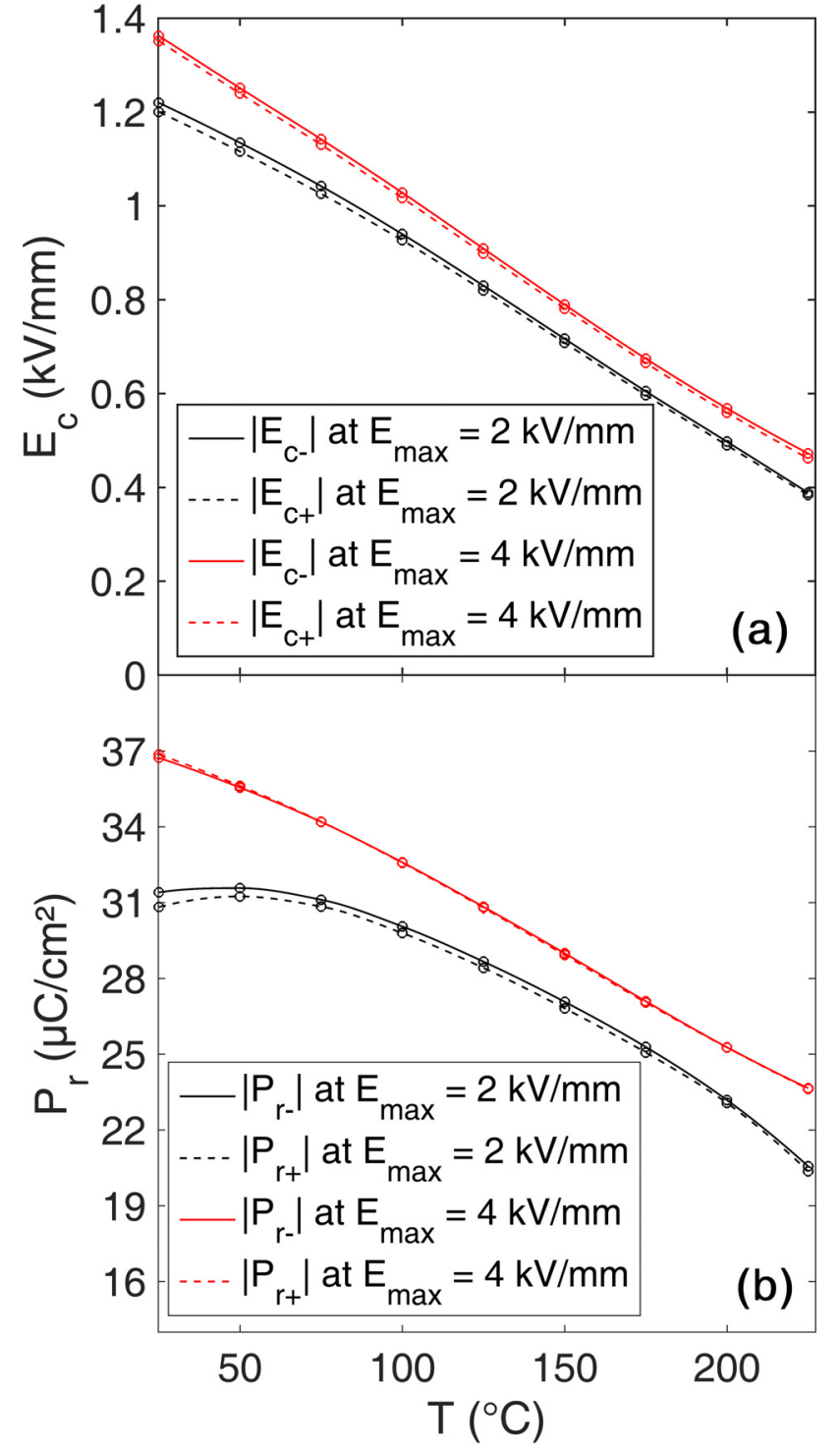

FIG. 5. Temperature dependence of the positive and negative coercive fields (a) and remanent polarizations (b). Graphs (a) and (b) were obtained from the macroscopic $P(E)$ hysteresis behavior $\left(f=0.1 \mathrm{~Hz}, E_{\max }=2 \mathrm{kV} / \mathrm{mm}\right.$, and $4 \mathrm{kV} / \mathrm{mm})$.

an approximately $1 \%$ difference of $P_{r}$ at room temperature (Fig. 5). These observations were expected since $2 \mathrm{kV} / \mathrm{mm}$ is not sufficient to reach the technical saturation. With an increasing temperature, however, the differences in the coercive field and remanent polarization at $2 \mathrm{kV} / \mathrm{mm}$ and $4 \mathrm{kV} /$ mm decrease.

The maximum polarization determined during measurement of the anhysteretic behavior is shown in Fig. 6(a). The maximum temperature for these measurements was $175^{\circ} \mathrm{C}$ (green data points) because the anhysteretic curve could not be measured above this temperature due to increased sample conductivity. The error induced by conductivity exceeded $10 \%$, which was not the case during bipolar loading that only required approximately $10 \mathrm{~s}$. Both the anhysteretic and the bipolar measurement methods are consistent; the maximum polarization decreases with an increasing temperature. For the anhysteretic measurement (green curve), a reduction of $9 \%$ is observed between room temperature and $175^{\circ} \mathrm{C}$.

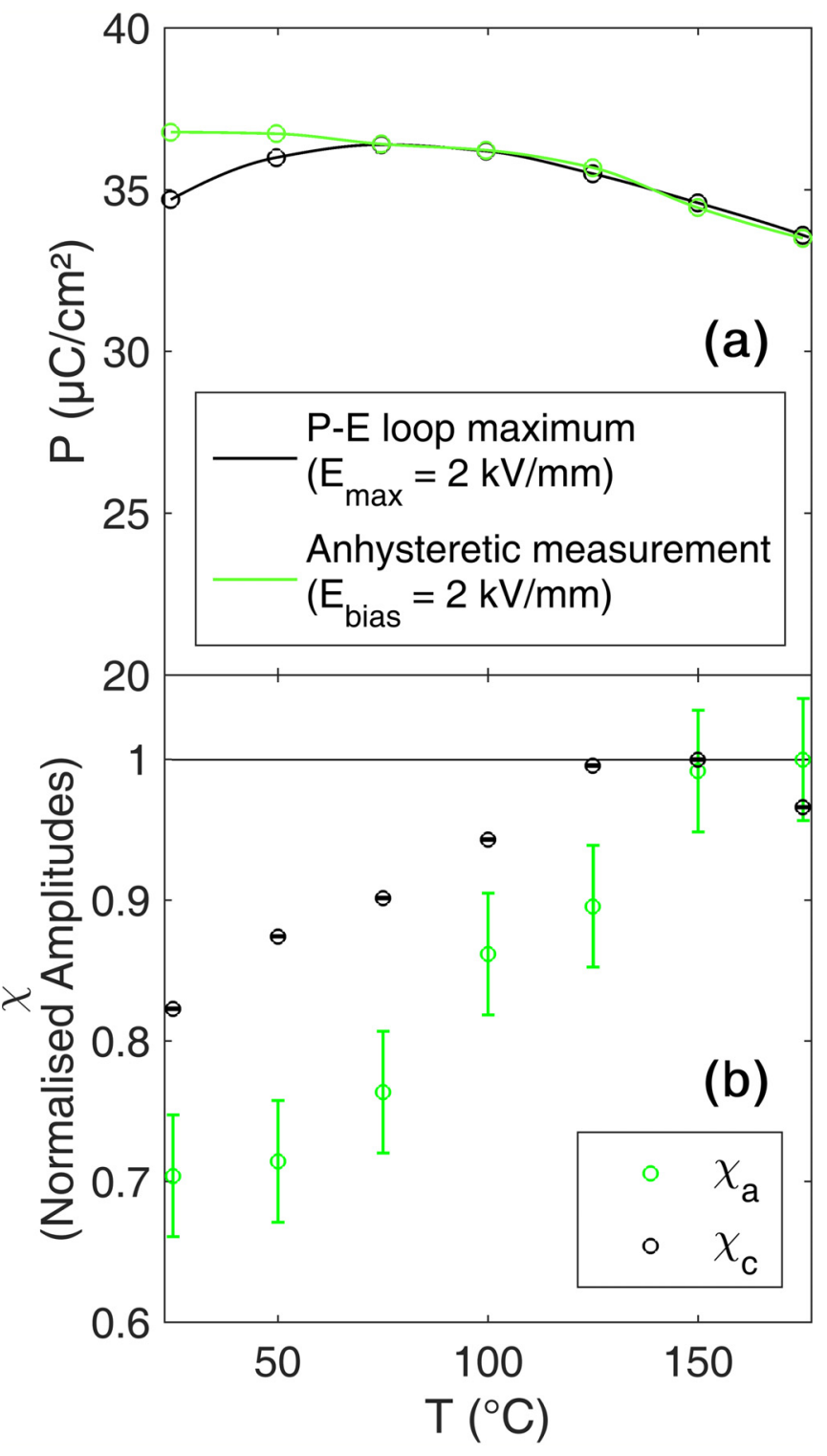

FIG. 6. (a) Maximum polarization value measured from a $P-E$ loop $\left(E_{\max }\right.$ $=2 \mathrm{kV} / \mathrm{mm}, f=0.1 \mathrm{~Hz}$, black curve) and from an anhysteretic measurement (green curve). (b) Temperature dependence of the normalised anhysteretic susceptibility $\chi_{a}$ and normalised differential susceptibility at coercive fields $\chi_{c} \cdot \chi_{a}$ is calculated from the anhysteretic curve (green dots-normalization factor of $4.80 \times 10^{5}$ ) and $\chi_{c}$ from a $4 \mathrm{kV} / \mathrm{mm} P-E$ loop (black dots-normalization factor of $1.03 \times 10^{5}$ ).

This is not a substantial decrease compared to the decrease in the remanent polarization, which was observed to be three times higher. Overall, the same polarization values are found for both methods, except at 25 and $50{ }^{\circ} \mathrm{C}$. At lower temperatures, the maximum electric field amplitude of $2 \mathrm{kV} / \mathrm{mm}$ is not high enough to fully saturate the material during bipolar loading, similar to observations of the differences in the remanent polarization during loading at $2 \mathrm{kV} / \mathrm{mm}$ and $4 \mathrm{kV} / \mathrm{mm}$ in Fig. 5(b). In contrast, the anhysteretic response is not affected since all anhysteretic points were measured with an $E_{\text {max }}$ of $4 \mathrm{kV} / \mathrm{mm}$ (the only changing parameter was $E_{\text {bias }}$ ).

To extract the dissipation contribution to the susceptibility, the evolution of $\chi_{a}$ is compared with the total susceptibility at the coercive field $(P=0)$. This susceptibility, namely $\chi_{c}$, corresponds to the slope of the $P-E$ loops at $E=E_{c}$. To 
improve the precision of $\chi_{c}$, the slopes at positive and negative coercive fields are averaged. The comparison is presented in Fig. 6(b). The data are given in normalized amplitudes, but the actual susceptibilities can be retrieved by multiplying the values by the normalization factors $(4.80$ $\times 10^{5}$ for $\chi_{a}$ and $1.03 \times 10^{5}$ for $\chi_{c}$ ). Unlike the other parameters, the susceptibilities $\chi_{a}$ and $\chi_{c}$ increase with an increasing temperature. The thermal energy enhances spontaneous polarization reorientation of crystal lattices in the presence of an external electric field, and this observation is consistent with other soft PZT studies. ${ }^{20}$ Susceptibility measured from $P-E$ loops is substantially lower than the anhysteretic susceptibility (ratio of 4 between $\chi_{a}$ and $\chi_{c}$ at $25^{\circ} \mathrm{C}$ ). This is expected since by definition the anhysteretic susceptibility is not affected by dissipation effects. Actually, the difference between $\chi_{a}$ and $\chi_{c}$ directly reflects the dissipative contribution to the macroscopic ferroelectric behavior. This relation can be described by separating the macroscopic behavior into two contributions, as introduced by Zhang et al. ${ }^{22}$ [see Eqs. (3)-(7)]:

$$
X(P, T)=X^{\text {int }}(P, T)+X^{\text {ext }}(P, T),
$$

where $X(P, T)$ is the total susceptibility, $X^{\text {int }}(P, T)$ is the intrinsic contribution to the macroscopic susceptibility, or volume contribution, and $X^{\text {ext }}(P, T)$ is the extrinsic contribution to the macroscopic susceptibility, or domain wall contribution. All contributions are functions of polarization and temperature. The extrinsic contribution can be further separated into two terms, related to a reversible domain wall contribution and to the dissipation contribution, respectively. The total susceptibility then becomes:

$$
X(P, T)=X^{\text {int }}(P, T)+X_{a}^{\text {ext }}(P, T)\left(1-\alpha_{d}(P, T)\right),
$$

where $X_{a}^{\text {ext }}(P, T)$ is the reversible domain wall contribution and $\alpha_{d}(P, T)$ is the dissipative or loss contribution coefficient. Here, the subscript "a" refers to "anhysteretic" and the total anhysteretic susceptibility $X_{a}(P, T)$ is:

$$
X_{a}(P, T)=X^{\text {int }}(P, T)+X_{a}^{\text {ext }}(P, T) .
$$

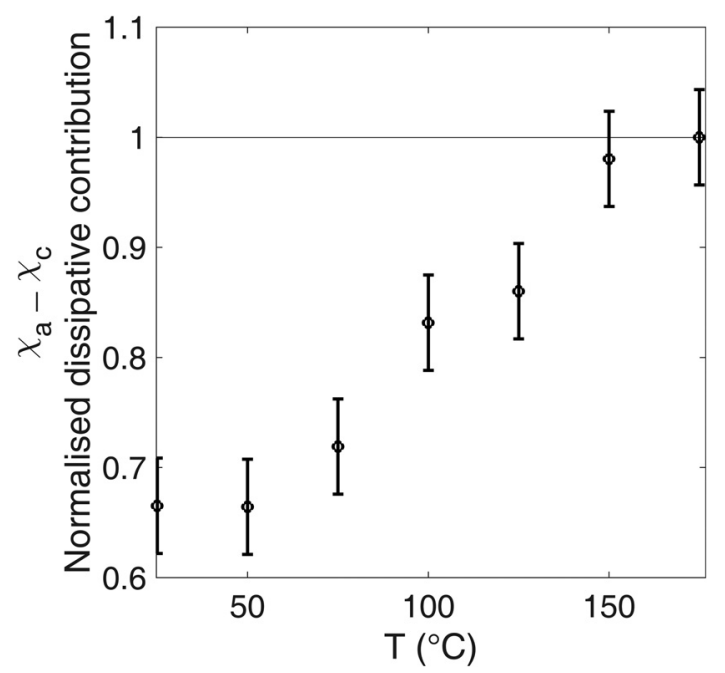

FIG. 7. Evolution of the normalized dissipative contribution to $\chi_{c}$ as a function of the temperature (normalization factor of $3.8 \times 10^{5}$ ).
From Eqs. (4) and (5), the dissipative contribution to the macroscopic susceptibility is expressed as:

$$
\alpha_{d} X_{a}^{\text {ext }}(P, T)=X_{a}(P, T)-X(P, T) .
$$

In this work, the measured anhysteretic susceptibility $\chi_{a}(T)$ [Fig. 6(b)] corresponds to $X_{a}(P=0, T)=X^{\text {int }}(P=0, T)$ $+X_{a}^{\text {ext }}(P=0, T)$. The coercive susceptibility $\chi_{c}(T)$ at $E=E_{c}$ is $X(P=0, T)=X^{\text {int }}(P=0, T)+X_{a}^{\text {ext }}(0, T) \cdot\left(1-\alpha_{d}\right.$ $(P=0, T))$. The dissipative contribution for $P=0$ is then:

$$
\alpha_{d} X_{a}^{\text {ext }}(P=0, T)=\chi_{a}(T)-\chi_{c}(T) .
$$

The evolution of this dissipative contribution is given as a function of temperature in Fig. 7. Values are normalized, but actual values can be retrieved with the normalization factor $3.8 \times 10^{5}$. The dissipative part contributing to $\chi_{c}$ increases by $35 \%$ from $25^{\circ} \mathrm{C}$ to $175^{\circ} \mathrm{C}$. This is more than the increase in $\chi_{a}$ for the same temperature range, which was found to be $+30 \%$ [Fig. 6(b)]. The dissipative contribution increases with temperature more than the reversible contribution. This is why the relative increase in $\chi_{\mathrm{c}}$ is only $18 \%$ [Fig. $6(\mathrm{~b})$ ] and not as high as that of $\chi_{\mathrm{a}}(30 \%)$.

Herbiet $e t ~ a l .{ }^{21}$ previously introduced such a three contribution separation. It was dedicated to dynamic loadings and based on a complex definition of the susceptibility. Here, Eq. (5) is a quasi-static formulation in which a standard loop measurement gives access to the total susceptibility $X(P, T)$. The anhysteretic curve then allows differentiation between the anhysteretic and dissipative contributions. Assuming that $X^{i n t}$ is isotropic and independent of the polarization level (linear dielectric behavior), an additional measurement at saturation allows an identification of $X^{\text {int }}$. Indeed at saturation the only contribution to polarization is the intrinsic effect. This allows for separation of the intrinsic contribution from the total anhysteretic behavior. Moreover, knowledge of $X^{\text {int }}$ allows determination of the dissipation coefficient $\alpha_{d}$ instead of the overall dissipation contribution $\alpha_{d} X_{a}^{\text {ext }}$. In the present case, $X^{\text {int }}$ could not be computed accurately due to the resolution in the data. Nevertheless, the anhysteretic curves are parallel at high electric fields (see Fig. 2), suggesting that $X^{\text {int }}$ is temperature independent. For temperatures near $25^{\circ} \mathrm{C}$, this is consistent with a previous study on $\mathrm{PZT}^{22}$ At higher temperatures, $X^{\text {int }}$ exponentially increases with temperature according to Herbiet et al. ${ }^{21}$ Future work involving precise computation of $X^{\text {int }}$ will allow further insight on the temperature dependence of the intrinsic effect.

\section{CONCLUSIONS}

In this work, the ferroelectric behavior of a co-doped PZT was studied using both classical loop measurements $(P-E$ and $S-E)$ and anhysteretic curves. This approach allowed for the separation of the reversible and irreversible contributions to the macroscopic behavior. Considering the reversible contribution, it was shown that an increase in temperature favors the polarizability of the material. The total susceptibility of the material also increases with temperature but not as significantly as the anhysteretic susceptibility. 
Indeed, the relative dissipative contribution to the susceptibility increases by $35 \%$ between 25 and $175^{\circ} \mathrm{C}$. It can then be interpreted that dissipation mechanisms are an impediment to the rise of susceptibility under increasing temperature. This is an important result for high temperature applications such as ferroelectric metal-oxide-semiconductor (MOS) transistor $^{31}$ where the susceptibility needs to be maximized. ${ }^{32}$ Even at temperatures around $150{ }^{\circ} \mathrm{C}$, this work shows that the susceptibility could be improved substantially by reducing the dissipative effects.

On the material characterization side, the anhysteretic curves, combined to $P-E$ and $S-E$ loops, can be used as an experimental assessment of the effect of external parameters on the mechanisms responsible for ferroelectric behavior. In this paper, the studied external parameter was the temperature, but the effect of stress, fatigue, or porosity could also be investigated using a similar technique. The access to mechanism separation can provide a powerful basis for the development and validation of micromechanical models for ferroelectric behavior.

\section{ACKNOWLEDGMENTS}

K.G.W. gratefully acknowledges the financial support from the Deutsche Forschungsgemeinschaft under WE4972/2. This work was also supported by the Automotive Mechatronics Chair, a co-operation between Faurecia, CentraleSupélec, and Esigelec.

\section{APPENDIX A: EFFECT OF CONDUCTIVITY ON ANHYSTERETIC MEASUREMENTS}

As specified in the paper, the electric displacement $D_{S}$ is obtained using a Sawyer-Tower circuit. This circuit integrates the current $I_{S}$ induced by the sample, which gives access to the electric charge $Q_{S}$. If the sample is a perfect insulator (infinite real impedance), the current $I_{S}$ would be equal to the displacement current $I_{D}$. These conditions cannot be reached experimentally. A conduction current $I_{C}$ due to material conductivity must be considered:

$$
I_{S}=I_{D}+I_{C} .
$$

This equation can be developed as:

$$
I_{S}=\iint\left(J_{D}+J_{C}\right) \cdot d S=\iint\left(J_{D}+\sigma \cdot E\right) \cdot d S,
$$

where $J_{D}$ is the displacement current density, $J_{C}$ is the conduction current density, $S$ the sample area (perpendicular to the electric field direction), $\sigma$ the sample conductivity, and $E$ the electric field applied to the sample.

As $I_{S}=\frac{\partial Q_{S}}{\partial t}$, a time integration of (A2) leads to electric charges:

$$
Q_{S}=\iiint J_{D} \cdot d S \cdot d t+S . \int \sigma \cdot E \cdot d t .
$$

The quantity $J_{D}$ is surface-independent and $\int J_{D} \cdot d t=D=P$ $+\varepsilon_{0} E$; thus:

$$
Q_{S}=S . D+S . \int \sigma \cdot E . d t,
$$

where $\varepsilon_{0}$ is the vacuum permittivity, $D$ the electric displacement, and $P$ the material polarization.
In terms of electric displacement, the equation can also be written:

$$
D_{S}=D+\int \sigma \cdot E \cdot d t
$$

The measured quantity $D_{S}$ is then equal to the electrical displacement $D$ (material behavior) plus a second quantity that depends on the material conductivity and the electric field. As $D$ is the variable of interest, the second quantity is a measurement error. This error must be either compensated or neglected if possible. In the present case, the error due to conductivity is studied for $E$ and $T$ (the temperature) constant over time. In these conditions, $\sigma$ is also constant over time which simplifies (A5):

$$
D_{S}=D+\sigma . E . t=D+C . t .
$$

The error due to conductivity is then a linear drift over time (with $C$, the slope coefficient of the drift). As $E$ is constant, $D$ becomes also constant for high values of $t$ (steady-state regime). In this condition, the drift coefficient $\mathrm{C}$ can be measured through a linear fitting of the data. Here, the sample is led to saturation during $60 \mathrm{~s}$ and then a linear fitting of $D(t)$ is done on 200 points to access the coefficient $C$. The measurement is repeated at different $E$ and $T$ to characterise the function $C(E, T)$. Results are shown in Fig. 8.

A measurement is considered reliable when the error induced on polarization due to conductivity is below $2 \%$ of the amplitude of the major loop. Similarly, the amplitude of polarization for the last cycle of the anhysteretic waveform is considered acceptable below $2 \%$ of the amplitude of the major loop [error introduced in point (iv) of page 4]. The value of the duration $t_{\max }$ is then the result of a compromise. For most experiments $\left(C<0.015 \mu \mathrm{C} / \mathrm{cm}^{2} \mathrm{~s}\right)$, the compromise is fulfilled by setting $t_{\max }$ at $60 \mathrm{~s}$. For high temperature, however, the conductivity is more influent, and $t_{\max }$ had to be decreased to keep in line with the error requirement. The exact values of $t_{\max }$ are given in Table I for each temperature and each bias electric field.

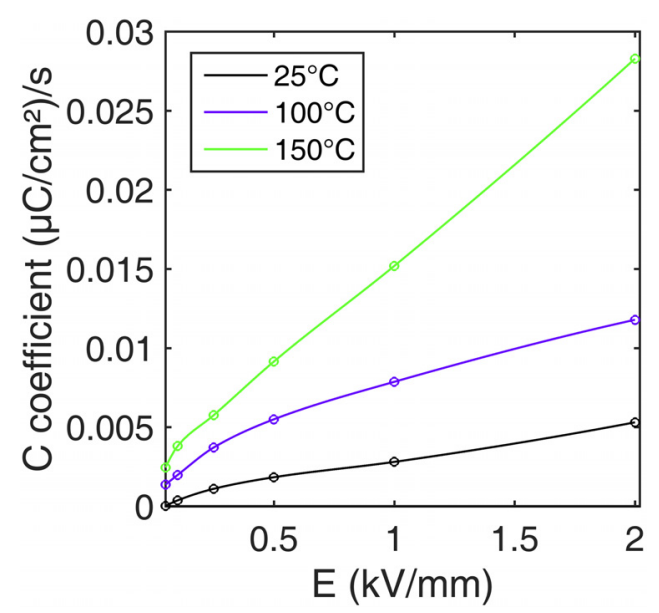

FIG. 8. Linear drift coefficient $C$ measured as a function of the electric field, for three steady temperatures. 
TABLE I. Parameter $t_{\max }$ used as a function of the temperature and the bias electric field. Values are given in seconds.

\begin{tabular}{lccccc}
\hline \hline & & \multicolumn{4}{c}{$E_{\text {bias }}(\mathrm{kV} / \mathrm{mm})$} \\
\cline { 3 - 5 } & & 0 & 0.5 & 1 & 2 \\
\hline Temperature $\left({ }^{\circ} \mathrm{C}\right)$ & 25 & 60 & 60 & 60 & 60 \\
& 50 & 60 & 60 & 60 & 60 \\
& 75 & 60 & 60 & 60 & 60 \\
& 100 & 60 & 60 & 60 & 60 \\
& 125 & 60 & 60 & 60 & 60 \\
& 150 & 60 & 50 & 40 & 30 \\
& 175 & 60 & 40 & 30 & 20 \\
\hline \hline
\end{tabular}

It must be noted that the error values given in the previous paragraph are overestimating the real measurement error as shown below. Assuming that $\sigma$ is independent from $E(t)$, Eq. (A5) gives:

$$
D_{S}=D+\sigma \int E . d t
$$

Case 1: $E(t)=2 \mathrm{kV} / \mathrm{mm}$

For $t_{\max }=60 \mathrm{~s}$, the integrated electric field $\int_{0}^{t_{\max }} E . d t$ is $120 \mathrm{kV} \mathrm{s} / \mathrm{mm}$.

Case 2: $E(t)$ not constant [see Eq. (1)]

The integral of $E(t)$ between 0 and $t_{\max }$ can be calculated using two consecutive integration by parts. Let denote $\mathrm{n}$, an integer representing the number of cycles in an anhysteretic measurement (with $n=f . t_{\max }$ ). The integral can be expressed as:

$$
\begin{gathered}
\int E . d t=\frac{n}{f} E_{\text {bias }}+\alpha\left(\frac{E_{\text {max }}}{1+k^{2}}-\frac{E_{\text {bias }}}{k}\right), \\
\text { where } \alpha=\frac{1-\exp (-2 k \pi n)}{2 \pi f} .
\end{gathered}
$$

Using $E_{\text {max }}=4 \mathrm{kV} / \mathrm{mm}, f=0.5 \mathrm{~Hz}, k=0.015$, and $E_{\text {bias }}$ $=2 \mathrm{kV} / \mathrm{mm}$, the integral of $E(t)$ gives $81.27 \mathrm{kV} \mathrm{s} / \mathrm{mm}$ : The ratio of integrals from both cases is 1.48 . Thus, the errors computed from Fig. 8 are overrating the error by $48 \%$, so that the effect of sample conductivity is even less important than estimated.

\footnotetext{
${ }^{1}$ A. Ayes, A. Maskay, and M. Pereira da Cunha, "Predicted and measured temperature compensated surface acoustic wave devices for hightemperature applications," Electron. Lett. 53(11), 699-700 (2017).

${ }^{2}$ R. R. Grzybowski, "High temperature passive components for commercial and military applications," in Proceedings of the 32nd Intersociety, Energy Conversion Engineering Conference (IEEE, 1997), Vol. 1, pp. 699-704.

${ }^{3}$ C. Randall, A. Kelnberger, G. Yang, R. E. Eitel, and T. Shrout, "High strain piezoelectric multilayer actuators-a material science and engineering challenge," J. Electroceram. 14(3), 177-191 (2005).

${ }^{4} \mathrm{M}$. Hooker, "Properties of PZT-based piezoelectric ceramics between - 150 and $250^{\circ}$ C," NASA/CR Report No. 208708, 1998.

${ }^{5}$ B. Kaeswurm, F. H. Schader, and K. G. Webber, "Ferroelectric, ferroelastic, piezoelectric, and dielectric properties of lead zirconate titanate from $-150^{\circ} \mathrm{C}$ to $350^{\circ} \mathrm{C}$," Ceram. Int. 44, 2358-2363 (2018).

${ }^{6} \mathrm{Y}$. Li, X. Zhou, and F. Li, "Temperature-dependent mechanical depolarization of ferroelectric ceramics," J. Phys. D: Appl. Phys. 43, 175501 (2010).

${ }^{7}$ M. Nicolai, S. Uhlig, A. Schönecker, and A. Michaelis, "Experimental investigation of non-linear behaviour of PZT piezoceramics at low temperatures," Adv. Sci. Technol. 56, 105-110 (2008).

${ }^{8}$ P. W. Forsbergh, Jr., "Piezoelectricity, Electrostriction and Ferroelectricity," in Encyclopedia of Physics/Handbuch der Physik Book Series (HDBPHYS) Dielectrics (1956), Vol. 4/17, pp. 264-392.
}

${ }^{9}$ A. Pramanick, D. Damjanovic, J. E. Daniels, J. C. Nino, and J. L. Jones, "Origins of electro-mechanical coupling in polycrystalline ferroelectrics during subcoercive electrical loading," J. Am. Ceram. Soc. 94(2), 293-309 (2011).

${ }^{10}$ B. Lewis, "Energy loss processes in ferroelectric ceramics," Proc. Phys. Soc. (London) 73, 17 (1959).

${ }^{11}$ J. Jones, E. Aksel, G. Tutuncu, T.-M. Usher, J. Chen, X. Xing, and A. Studer, "Domain wall and interphase boundary motion in a two-phase morphotropic phase boundary ferroelectric: Frequency dispersion and contribution to piezoelectric and dielectric properties," Phys. Rev. B 86(3), 024104 (2012).

${ }^{12} \mathrm{H}$. Fu and R. Cohen, "Polarization rotation mechanism for ultrahigh electromechanical response in single-crystal piezoelectrics," Nature 403, 281-283 (2000).

${ }^{13}$ D. Damjanovic, "Stress and frequency dependence of the direct piezoelectric effect in ferroelectric ceramics," J. Appl. Phys. 82, 1788 (1997).

${ }^{14}$ R. Eitel, T. Shrout, and C. Randall, "Nonlinear contributions to the dielectric permittivity and converse piezoelectric coefficient in piezoelectric ceramics," J. Appl. Phys. 99, 124110 (2006).

${ }^{15}$ D. Damjanovic, "Hysteresis in piezoelectric and ferroelectric materials," in The Science of Hysteresis (Elsevier, 2006), Vol. 3, pp. 337-452.

${ }^{16}$ R. E. Eitel, "Rayleigh law response in ferroelectric ceramics quantifying domain wall dynamics and structural relationships," in Sixteenth IEEE International Symposium on Applications of Ferroelectrics, ISAF 2007, 2007.

${ }^{17}$ B. Kaeswurm, V. Segouin, L. Daniel, and K. G. Webber, "The anhysteretic polarisation of ferroelectrics,” J. Phys. D: Appl. Phys. 51, 075305 (2018).

${ }^{18}$ R. Bozorth, Ferromagnetism (Van Nostrand, 1951).

${ }^{19}$ L. Daniel, D. Hall, and P. J. Withers, "A multiscale model for reversible ferroelectric behaviour of polycrystalline ceramics," Mech. Mater. 71, 85-100 (2014).

${ }^{20}$ D. Hall, "Nonlinearity in piezoelectric ceramics," J. Mater. Sci. 36, 4575-4601 (2001).

${ }^{21}$ R. Herbiet, U. Robels, H. Dederichs, and G. Arlt, "Domain wall and volume contributions to material properties of PZT ceramics," Ferroelectrics 98, 107-121 (1989).

${ }^{22}$ Q. M. Zhang, H. Wang, N. Kim, and L. E. Cross, "Direct evaluation of domain wall and intrinsic contributions to the dielectric and piezoelectric response and their temperature dependence on lead zirconate titanate ceramics," J. Appl. Phys. 75, 454 (1994).

${ }^{23}$ F. H. Schader, G. Rossetti, Jr., J. Luo, and K. G. Webber, "Piezoelectric and ferroelectric properties of $\langle 001\rangle_{\mathrm{C}} \mathrm{Pb}\left(\mathrm{In}_{1 / 2} \mathrm{Nb}_{1 / 2}\right) \mathrm{O}_{3}-\mathrm{Pb}\left(\mathrm{Mg}_{1 / 3} \mathrm{Nb}_{2 / 3}\right) \mathrm{O}_{3}$ $-\mathrm{PbTiO}_{3}$ single crystals under combined thermal and mechanical loading," Acta Mater. 126, 174-181 (2017).

${ }^{24}$ D. Damjanovic, M. Budimir, M. Davis, and N. Setter, "Monodomain versus poly-domain piezoelectric response of $0.67 \mathrm{~Pb}\left(\mathrm{Mg} \_1 / 3 \mathrm{Nb}_{2 / 3}\right) \mathrm{O} \_3$ $0.33 \mathrm{PbTiO}_{3}$ single crystals along nonpolar directions," Appl. Phys. Lett. 83, 527 (2003).

${ }^{25}$ M. Stewart, M. G. Cain, and D. Hall, Ferroelectric Hysteresis Measurement and Analysis (National Physical Laboratory and University of Manchester, 1999), ISBN: 1368-6550.

${ }^{26}$ D. Bochenek, R. Skulski, P. Wawrzala, and D. Brzezinska, "Dielectric and ferroelectric properties and electric conductivity of sol gel derived PBZT ceramics," J. Alloys Compd. 509, 5356-5363 (2011).

${ }^{27}$ A. B. Kounga, T. Granzow, E. Aulbach, M. Hinterstein, and J. Rödel, "High-temperature poling of ferroelectrics," J. Appl. Phys. 104, 024116 (2008).

${ }^{28} \mathrm{H}$. Kungl and M. J. Hoffmann, "Temperature dependence of poling strain and strain under high electric fields in LaSr-doped morphotropic PZT and its relation to changes in structural characteristics," Acta Mater. 55, 5780-5791 (2007).

${ }^{29}$ P. M. Weaver, M. G. Cain, and M. Stewart, "Temperature dependence of high field electromechanical coupling in ferroelectric ceramics," J. Phys. D: Appl. Phys. 43, 165404 (2010).

${ }^{30}$ A. Q. Jiang and T. A. Tang, "Remanent polarization reduction with enhanced temperature in ferroelectric thin films," J. Appl. Phys. 104, 024104 (2008).

${ }^{31}$ G. A. Salvatore, L. Lattanzio, D. Bouvet, I. Stolichnov, N. Setter, and A. M. Ionescu, "Ferroelectric transistors with improved characteristics at high temperature," Appl. Phys. Lett. 97, 053503 (2010).

${ }^{32}$ J. Robertson, "High dielectric constant gate oxides for metal oxide Si transistors," Rep. Prog. Phys. 69, 327-396 (2006). 